\title{
Emerging treatments for postmenopausal osteoporosis - focus on denosumab
}

REVIEW

This article was published in the following Dove Press journal:

Clinical Interventions in Aging

29 May 2009

Number of times this article has been viewed

\section{Piet Geusens}

Department of Internal Medicine, Subdivision of Rheumatology, Maastricht University Medical Center, Maastricht, The Netherlands and Biomedical Research Institute, University Hasselt, Belgium
Correspondence: Piet Geusens

Department of Internal Medicine, Subdivision of Rheumatology, Maastricht University Medical Center, Maastricht,

The Netherlands and Biomedical

Research Institute, University Hasselt, Belgium

Tel +32 89362977

$\mathrm{Fax}+3289304186$

Email piet.geusens@scarlet.be

\begin{abstract}
The pathway of the receptor activator of the nuclear factor $\kappa B$ ligand (RANKL), RANK and osteoprotegerin (OPG) plays a central role in coupling bone formation and resorption during normal bone turnover and in a wide spectrum of diseases characterized by disturbed bone remodeling, increased bone resorption and bone destruction (osteoporosis, Paget's disease of bone, rheumatoid arthritis [RA], metastatic bone disease). Clinical trials indicate that denosumab, a RANKL-specific recombinant humanized monoclonal antibody, is effective in suppressing bone resorption, resulting in increase in bone mineral density (BMD) in postmenopausal women with low BMD, and has the potential to prevent progression of erosions in RA and of skeletal-related events in metastatic bone disease. The effects on fracture reduction in postmenopausal osteoporosis are awaited from the recently finished FREEDOM study. In clinical trials with denosumab, overall adverse events were similar to placebo or comparators, indicating a favorable safety profile in these diseases, which until now have been available up to 4 years, but data on long-term safety will be needed.
\end{abstract}

Keywords: denosumab, postmenopausal osteoporosis, bone mineral density

\section{Introduction}

Bone's resistance to fracture is determined by its structural and material characteristics, which are determined by life-long remodeling of bone by osteoclastic bone resorption and osteoblastic bone formation. ${ }^{1}$ The discovery of the receptor activator of nuclear factor $\kappa \mathrm{B}$ ligand (RANKL), osteoprotegerin (OPG) and RANK is a major contribution in our understanding of the balanced coupling in space, time and quantity between bone formation and resorption and its disconnection in several bone diseases characterized by increased bone resorption and destruction, such as osteoporosis, rheumatoid arthritis (RA), Paget's disease of bone and metastatic bone disease. ${ }^{2}$

In this review I focus on the clinical relevance of the RANKL/RANK/OPG pathway in health and disease and the effect of denosumab, a recombinant fully humanized monoclonal RANKL-specific antibody that inhibits bone resorption, in a wide spectrum of diseases. For an extensive list of references on preclinical studies, the reader is referred to a recent in-depth review. ${ }^{2}$

\section{The RANKL/OPG/RANK pathway: the discovery of how bone formation and resorption are coupled}

For many decades, it has been known that bone resorption and formation in healthy adults are coupled in space, time and quantity, ${ }^{3}$ but the exact mechanisms behind this coupling remained for a long time obscure. ${ }^{4}$ In the late 1990s, the first component identified for a 
novel pathway regulating bone remodeling was OPG. ${ }^{5,6} \mathrm{OPG}$ was discovered by sequencing random clones from a cDNA library and its gene was shown to encode a novel member of the tumor necrosing factor (TNF) receptor family. Overexpression of the OPG gene in mice resulted in high bone mass and marked reduction in osteoclast number and activity, while OPG knockout (KO) mice had low BMD, increased numbers of osteoclasts, had more woven bone, and developed spontaneous fractures. ${ }^{7}$ OPG is a decoy receptor for RANKL and in this way decreases bone resorption. OPG is produced by osteoblasts, endothelial cells, vascular smooth muscle and other cells.

Already in 1980 it was suggested that osteoblasts might be involved in osteoclastogenesis. ${ }^{8}$ The nature of this hypothesized 'osteoclast activating factor' remained elusive, until 1998, when several laboratories independently identified RANKL as a new member of the TNF family of transmembrane and soluble ligands that could bind to $\mathrm{OPG}^{9,10}$ and which was one year earlier identified as a product of activated $T$ cells that promotes dentritic cell survival and co-stimulation. ${ }^{11}$ RANKL KO mice have high bone mass and virtually no osteoclasts. RANKL binds to RANK and is in this way involved in numerous aspects of osteoclast differentiation and function (fusion, differentiation, attachment to bone, activation and survival). In most instances RANKL relies on macrophagecolony stimulating factor (M-CSF) as a cofactor for osteoclast differentiation, but RANKL can stimulate osteoclastogenesis and bone resorption in mice lacking functional M-CSF and no factor or combination of factors have been shown to be capable of restoring bone resorption when RANKL is absent, indicating the dominant role of RANKL in the regulation of bone resorption. ${ }^{2}$ RANKL is produced by cells of the osteoblastic lineage and by activated T cells.

RANK is a cell membrane receptor of the TNF family to which RANKL is bound and is found on dendritic cells and cells of the osteoclast lineage. RANK KO in mice results in the same phenotype as RANKL KO with high bone mass and virtually no osteoclasts.

The activation of RANK in osteoclasts results in activation of several intracellular signal transduction pathways, which bind the nuclear factor $\kappa \mathrm{B}(\mathrm{NF} \kappa \mathrm{B})$. After ubiquitination of signal transducers (which results in their degradation by proteasomes), NF $\kappa \mathrm{B}$ is released, so that it can translocate to the nucleus, where it upregulates cofactors that induce osteoclastogenic gene transcription (Figure 1).

The many available data suggest that the RANKL:OPG ratio represents an important determinant of bone resorption. ${ }^{2}$ Numerous growth factors, hormones, cytokines and drugs that influence bone turnover have been shown to influence the expression of RANKL and OPG. ${ }^{2}$ The RANKL:OPG ratio is decreased by estrogens and increased by glucocorticoids (GC), parathyroid hormone (PTH), PTH-related protein (PTHrP) and prostaglandins. Other molecules and agents that regulate RANKL and/or OPG and that are proresorptive include interleukin-1 (IL-1), IL-17, TNF- $\alpha$, 1,25-dihydroxyvitamin $\mathrm{D}$ and bone morphogenetic protein 2 (BMP-2). Those which suppress osteoclastogenesis and regulate RANKL and/or OPG are IL-4, IL-13, interferon- $\gamma$ $(\mathrm{IFN}-\gamma)$ and transforming growth factor- $\beta$ (TGF- $\beta$ ).

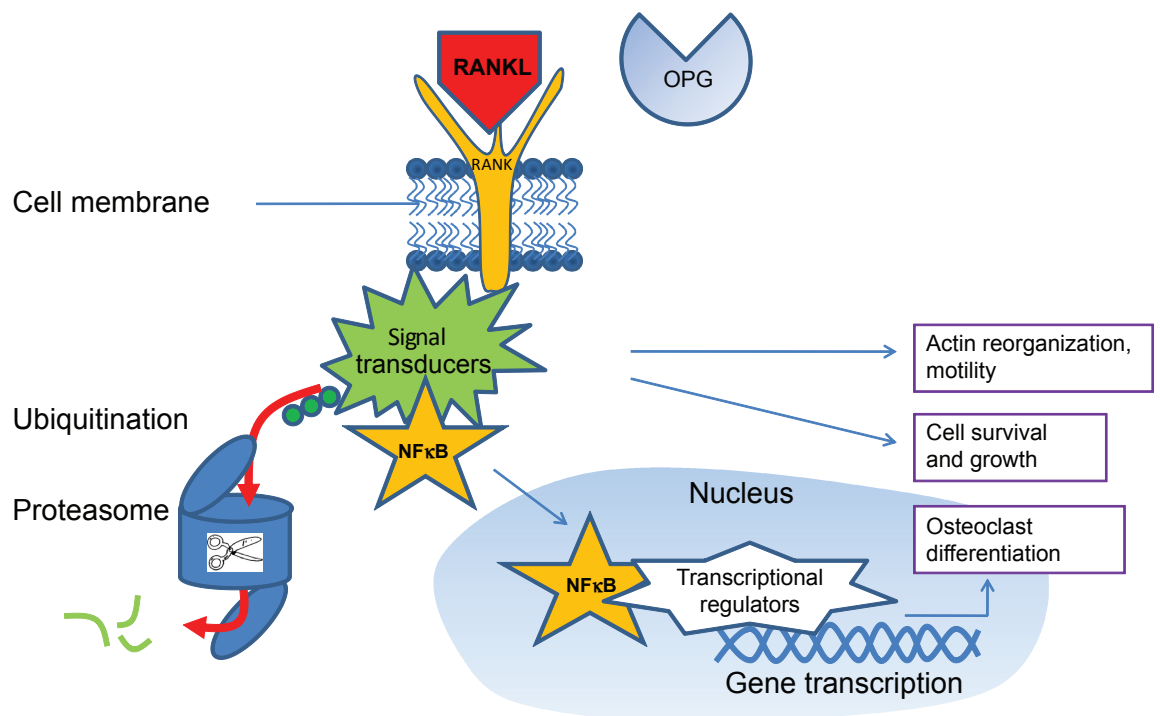

Figure I Free RANKL (ie, not bound by osteoprotegerin [OPG]) binds to the transmembrane RANK receptor, which upregulates intracellular signal transducers which are involved in cytoskeletal organization, cell motility, growth and survival, and some also bind NF $\kappa$ B. After ubiquitination, signal transducers are released from $N F \kappa B$ and degraded by proteasomes. NF $K B$ can than migrate to the nucleus, were it upregulates transcriptional regulators that start osteoclastogenic gene transcription. ${ }^{2}$ 
OPG prevents the ability of proresorptive agents (IL-1, TNF- $\alpha$, PTH, PTHrP, vitamin D) to increase bone resorption in mice. ${ }^{2}$ In RANK KO mice, IL-1 and TNF- $\alpha$ induced the appearance of few if any osteoclasts with no evidence of bone resorption. TNF- $\alpha$ was shown to require very low 'permissive' levels of RANKL to stimulate osteoclasts. Thus, whereas TNF- $\alpha$, IL-1 and other molecules have certain independent stimulatory effects on osteoclasts, the very existence of those osteoclasts remains dependent on RANKL/RANK signaling. ${ }^{2}$

The Wnt (wingless) signaling in osteoblasts is also a source of regulation of OPG. Ablation of the intracellular canonic signaling pathway ( $\beta$-catenin) in osteoblasts resulted in reduced OPG expression and bone loss, while its over expression resulted in increased OPG expression and high bone mass. ${ }^{12}$ Ablation of osteocytes in mice increased bone resorption and the RANKL:OPG ratio. ${ }^{13}$ These observations have enabled to integrate the RANKL/RANK/OPG and Wnt signaling pathways in understanding normal and diseased bone. ${ }^{14}$

\section{The RANKL/OPG/RANK pathway: its role in healthy bone turnover}

The dominant physiological role of the RANKL/RANK/OPG pathway in the regulation of bone remodeling has been demonstrated in the above mentioned transgenic and $\mathrm{KO}$ mice models.

Injection of recombinant RANKL and OPG in mice induced a rapid and marked effect on bone turnover markers (BTM) and number of osteoclasts. One RANKL injection stimulated bone resorption within 1 hour. ${ }^{15}$ One OPG injection resulted in decreased BTM within 2 hours and a $50 \%$ to $60 \%$ reduction in osteoclast numbers within 12 to 24 hours with a long lasting effect which is fully reversible after 1 to 2 months, and which is the result of the long half life and the time required to generate new osteoclasts once the drug is cleared. ${ }^{16}$ In small animals, OPG had positive effects on bone mineral content (BMC) and BMD, trabecular bone volume (TBV) and bone strength. ${ }^{2}$ Similar positive effects were found with denosumab in monkeys. Bone strength parameters were strongly correlated with bone mass parameters, suggesting that RANKL inhibition improved bone strength primarily by increasing bone mass and that bone formed during antiRANKL treatment has normal material properties. RANKL inhibition in animals also prevented bone loss after ovariectomy, orchidectomy, GC use, and disuse. ${ }^{2}$

The discovery of the RANKL/OPG/RANK pathway and these experiments have elucidated our understanding of cellular and mechanisms of bone remodeling. During normal bone remodeling, osteoclast precursors derived from the circulation or bone marrow develop a RANKL/RANK driven cell contact with osteoblasts. This enables osteoclast precursors to differentiate to mature osteoclasts and activates their bone resorption capacity by attaching to bone, polarizing, becoming mobile, expressing the calcitonin receptor and tartrate-resistant alkaline phosphatase (TRAP), producing $\mathrm{H}^{+}$by carbonic anhydrase II, secretion of $\mathrm{H}^{+}$by the osteoclast-specific vacuolar ATP-ase pump and $\mathrm{Cl}^{-}$by the $\mathrm{Cl}^{-}$channel to form HCL that degrades bone mineral, lysosomal release of cathepsin-K and matrix metalloproteinases (MMPs) that degrade bone matrix proteins, and by contributing to osteoclast mobility and survival. ${ }^{17}$

\section{Non-osseous effects of the RANKL/ OPG/RANK pathway}

The above-mentioned OPG, RANKL and RANK KO animal models revealed significant extraskeletal manifestations with regard to vascular calcifications, the immune system and mammary gland development.

OPG KO animals developed medial calcification of the aorta and renal arteries, ${ }^{7}$ which could be prevented by transgenic over expression of soluble OPG. RANKL and OPG have been identified in human atherosclerotic plaques. ${ }^{18}$ The mechanism by which OPG could serve a protective role in vascular disease is unclear. Experiments in animal models of atherosclerosis indicate that the prevention of vascular calcification is probably related to the suppression of bone resorption, with neutral effects on existing atherosclerotic changes. ${ }^{2}$

RANK and RANKL KO results in lymph node agenesis, indicating the role of the RANKL/RANK/OPG pathway development and function of the immune system. ${ }^{2}$ As mentioned earlier, activated $\mathrm{T}$ cells produce RANKL and thereby regulate the function and survival of dendritic cells. ${ }^{11}$ However, RANKL inhibition did not lead to dendritic cell suppression, probably due to the central role of CD40 cross-talk between $\mathrm{T}$ cells and dendritic cells, which compensates for the total absence of RANKL/RANK in KO mice. ${ }^{2}$ RANKL and RANK are found in bone erosions of RA, rendering RANKL a possible target to prevent bone erosions in RA.

RANKL/RANK KO mice have a defect in mammary gland development during pregnancy and lactation. ${ }^{2}$ However, OPG transgenic mice did not show failure of lactation, while bone resorption was inhibited. ${ }^{2}$

\section{The RANKL/OPG/RANK pathway in osteolytic and destructive bone diseases}

The RANKL/OPG/RANK pathway plays a crucial and consistent role in a wide spectrum of diseases of bone 
characterized by increased bone resorption, disturbed coupling between bone formation and resorption, and bone destruction.

Mutations of the genes for RANKL, OPG and intracellular signal transducers of NF $\mathrm{KB}$ have been identified in diseases characterized by focal disturbed bone remodeling, such as juvenile and classical Paget's disease of bone, familial expansile osteolysis, expansile skeletal hyperphosphatasia and inclusion body myopathy combined with Paget's disease and frontotemporal demantion (IBMPFD). ${ }^{19}$

Rheumatoid arthritis (RA) is characterized by joint inflammation and bone erosions, periarticular and generalized bone loss. Bone erosions were already described in 1878 as 'caries of the joints, ${ }^{, 20}$ but only during the last decades it has been shown that these erosions contain multinucleated cells which were identified as osteoclasts. ${ }^{21-23}$ Animal models of RA (collagen-induced arthritis (CIA), adjuvant-induced arthritis (AIA) and TNF-mediated arthritis) indicated early upregulation of RANKL and suppression of OPG in inflamed joints. RANKL KO and OPG prevented formation of erosions, without effect on inflammation. ${ }^{2}$ This indicates that bone destruction and inflammation can be dissociated on the basis of specific inhibition of the RANKL/RANK pathway. OPG also prevented periarticular and generalized bone loss in such animal models ${ }^{2}$ and preserved cartilage in several arthritic animal models. ${ }^{2}$ RANKL, RANK and OPG are expressed in human articular cartilage, but their functional roles have not been clearly demonstrated, and RANK is probably inactive in chondrocytes. ${ }^{2}$ A likely mechanism for chondroprotection by OPG is to preserve subchondral bone against subchondral osteoclast invasion. ${ }^{2}$

More than 100 years ago it had been observed that bone was a fertile soil for the localization and growth of particular types of cancer cells. ${ }^{24}$ Recently it has become clear that there is a bidirectional communication between tumor cells and bone cells, resulting in a vicious circle of bone destruction and tumor growth. ${ }^{2,25,26}$ In metastatic bone disease, cancer cells are able to increase the RANKL: OPG ratio directly or by the help of T-cells, osteoblast/stromal cells and endothelial cells, along with the production of other osteoclast mediators such as PTHrP. This stimulates osteoclasts to remove bone, enabling cancer cells to make space for further growth within bone, ${ }^{25,26}$ and releasing growth factors from bone that further stimulate tumor growth. Multiple myeloma cells are even more destructive for bone, as they release not only RANKL, but also dickkopff(DKK), which suppresses bone formation, ampliflying in this way room for tumor growth. ${ }^{26}$

\section{Denosumab: the effect of RANKL inhibition in osteoporosis, RA and metastatic bone disease}

Denosumab is a fully human monoclonal antibody with a high affinity and specificity for RANKL and binds and neutralizes the activity of human RANKL in a similar fashion to the action of OPG. ${ }^{2}$ Denosumab does not cross-react with TNF- $\alpha$, TNF- $\beta$, TNF-related apoptosis-inducing ligand (TRAIL), or CD40 ligand.

\section{Postmenopausal osteoporosis}

The first clinical study on RANKL inhibition was performed in postmenopausal women with BMD-osteoporosis using OPG bound to the constant fragment $(\mathrm{Fc})$ of human IgG1 (Fc-OPG), which showed a dose-dependent decrease of markers of bone resorption within 4 days with a maximum decrease of $80 \%$ with the highest dose and lasting 45 days, without changes in markers of bone formation. ${ }^{27}$ However, Fc-OPG was not further studied because denosumab was superior in reducing bone resorption at lower dose and with longer duration, ${ }^{24}$ antibodies to Fc-OPG could be raised ${ }^{24}$ and because of the concern that Fc-OPG could bind to TRAIL, which could inhibit its role in tumor surveillance. ${ }^{28}$

Denosumab has been studied in postmenopausal women in the context of its effect on bone remodeling, BMD, parameters of bone strength in the hip region, and safety.

In a randomized, placebo-controlled phase- 1 study in 49 healthy postmenopausal women, a single SC injection of denosumab caused a dose-dependent rapid (within 12 hours), profound (up to 84\%) and sustained (up to 6 months) decrease in bone resorption (reflected by urinary N-telopeptide (NTX)) and was well tolerated. ${ }^{28}$ Bone formation (as reflected by bone-specific alkaline phosphatase (BALP)) did not decrease remarkably until after 1 month, indicating that the effect of denosumab is primarily antiresorptive. Intact PTH levels increased up to 3 -fold after 4 days, but returned towards baseline with follow-up. This study indicated that denosumab effectively decreases bone resorption in humans and that long intervals between doses of denosumab might be possible.

Efficacy and safety of denosumab were evaluated in a randomized, placebo-controlled, dose-ranging phase 2 study in 412 postmenopausal women with low BMD in the spine or femur and included a group of patients treated with open-label alendronate $70 \mathrm{mg}$ /week..$^{29}$ Denosumab (at a dose of 6,14 or $30 \mathrm{mg}$ every 3 months or 14, 60, 100 or $210 \mathrm{mg}$ every 6 months over a period of 12 months) resulted in a rapid and sustained effect on BTM and a rapid increase in 
BMD in the spine, hip and distal radius, which was superior to placebo and similar or greater (for changes in BMD in the total hip and in the distal radius and in markers of bone resorption) than with weekly $70 \mathrm{mg}$ of alendronate (Figure 2). These changes were sustained in an extension study over 24 months (Figure 3). ${ }^{30}$ Adverse events (AE) and serious AE (SAEs) were similar in character and percentage with denosumab compared with placebo. In terms of increase in BMD, the dose of $30 \mathrm{mg} / 3$ months and $60 \mathrm{mg} / 6$ months appeared optimal.

In a post hoc analysis of this study in a subgroup of 116 patients treated with denosumab 60 mg every 6 months, weekly alendronate $70 \mathrm{mg}$ or placebo, structural geometry of the hip was evaluated on DXA images, and derived strength indices were calculated, including bone cross-sectional area, section modulus and buckling ratio. ${ }^{31}$ At 12 and 24 months denosumab and alendronate improved these parameters compared with placebo. Denosumab effects were greater than alendronate at the intertrochanteric and femoral shaft sites. These results suggested that denosumab treatment may lead to improved bone biomechanical properties, similar or greater than with weekly alendronate.

The phase 2 study was continued for an additional 24 months to study the long-term efficacy and safety of denosumab and the effects of discontinuing and restarting different doses of denosumab treatment. ${ }^{32}$ With continuous treatment, denosumab led to further gains in BMD at the lumbar spine, total hip and distal $1 / 3$ radius and sustained reduction in BTM throughout the course of the study. The effects on BTM were fully reversible with discontinuation and restored with subsequent retreatment (Figure 4).
In a double-blind phase 3 study, including 1180 postmenopausal women with low BMD (T-score $\leq 2.0$ in the lumbar spine or total hip), the efficacy and safety of denosumab $60 \mathrm{mg}$ every 6 months and alendronate $70 \mathrm{mg}$ weekly during 12 months treatment were compared. ${ }^{33}$ At the total hip, denosumab significantly increased BMD compared to alendronate $(2.5 \%$ versus $2.6 \%, \mathrm{p}<0.0001)$. Furthermore, significantly greater increases in BMD were observed with denosumab at all measured skeletal sites (12-month treatment difference: $1.1 \%$ in the lumbar spine, $0.6 \%$ in the femoral neck, $1.0 \%$ in the trochanter and $0.6 \%$ in the $1 / 3$ radius; $p<0.001$ at all sites). Compared to alendronate, treatment with denosumab resulted in greater reductions in BTM (serum C-telopeptide-1 [sCTX1] for bone resorption and P1NP for bone formation) at each time point assessed through month 9 for sCTX1 and month 12 for P1NP. Maximum reduction of $\mathrm{P} 1 \mathrm{NP}$ in the alendronate group was reached at month 3 and remained constant throughout the study (Figure 5). In contrast, maximal reduction of SCTX1 in the denosumab group was observed at 1 month, with attenuated reduction in $\mathrm{SCTX} 1$ at the end of the 6-month dosing interval. AEs were similar for denosumab- and alendronatetreated subjects. SAEs were similar between denosumab and alendronate for infections $(1.5 \%$ versus $1.0 \%, \mathrm{p}=0.61)$ and malignant neoplasm $(1.0 \%$ versus $0.9 \%, \mathrm{p}=1.00)$.

Similar changes in BMD and BTM were reported in a 2-year randomized, double-blind, placebo-controlled study in 332 postmenopausal women with BMD-osteopenia treated with the same dose of denosumab (60 mg every 6 month). ${ }^{34}$ SEAs for infections were significantly higher in the denosumab group $(4.9 \%)$ then with placebo $(0.6 \%, \mathrm{p}<0.05)$ and

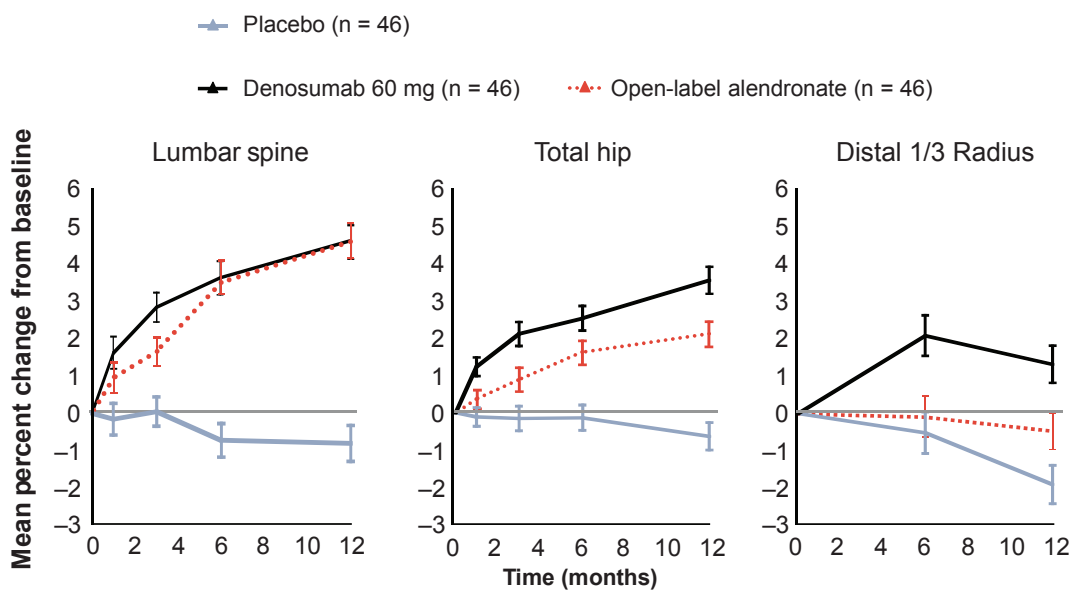

Figure 2 Phase 2 study of denosumab every 6 months in postmenopausal women with low (bone mineral density) BMD: lumbar spine, total hip, and distal I/3 radius BMD at 12 months. Adapted from J Bone Miner Res. 2007;22:1832-184I, ${ }^{30}$ with permission of the American Society for Bone and Mineral Research; and from N Engl J Med. 2006;354:82I-83I. ${ }^{29}$ 


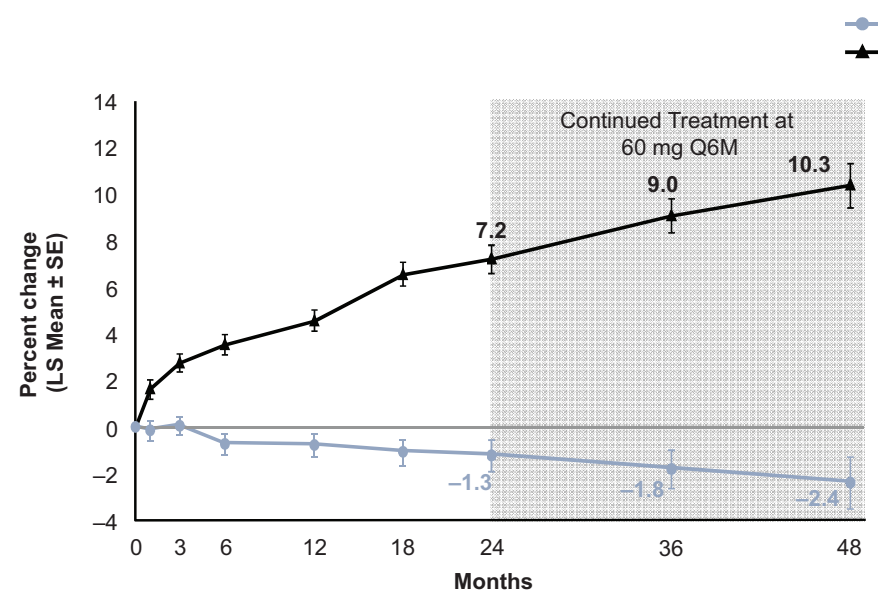

Figure 3 Effect of 4 years of denosumab every 6 months on lumbar spine (LS) bone mineral density. Adapted from J Bone Miner Res. 2007;22:I832-184I, ${ }^{30}$ with permission of the American Society for Bone and Mineral Research.

similar for neoplasm $(2.4 \%$ with denosumab versus $0.6 \%$ with placebo, $\mathrm{p}=0.215$ ).

Meanwhile, the pivotal fracture trial (Fracture REduction Evaluation of Denosumab in Osteoporosis every 6 Months, the 'FREEDOM' study) with fracture reduction as a primary endpoint has been accomplished in 7,808 postmenopausal women with BMD-osteoporosis, ${ }^{35}$ and a peer-reviewed publication is expected soon.

\section{Rheumatoid arthritis}

In animal models of arthritis (CIA and AIA) RANKL expression has been demonstrated within 1 to 14 days of the appearance of joint inflammation. ${ }^{2}$ OPG fully prevented the occurrence of bone erosions, without effect on cartilage loss and inflammation. In patients with RA, RANKL expression has been documented in erosions. Furthermore, baseline serum levels of the RANKL:OPG ratio predicted future joint damage independent of other predictors of joint damage in patients with early untreated RA. ${ }^{36}$

In a placebo-controlled study in 227 patients with RA taking methotrexate, 71 were treated with denosumab $60 \mathrm{mg}$, 72 with $180 \mathrm{mg}$ denosumab and 75 had placebo injections every 6 months during 12 months. ${ }^{37}$ At 6 months, the increase in MRI erosion score from baseline was significant lower in the 180-mg denosumab group than in the placebo group ( 0.06 versus $1.75, p=0.007)$. A significant difference in
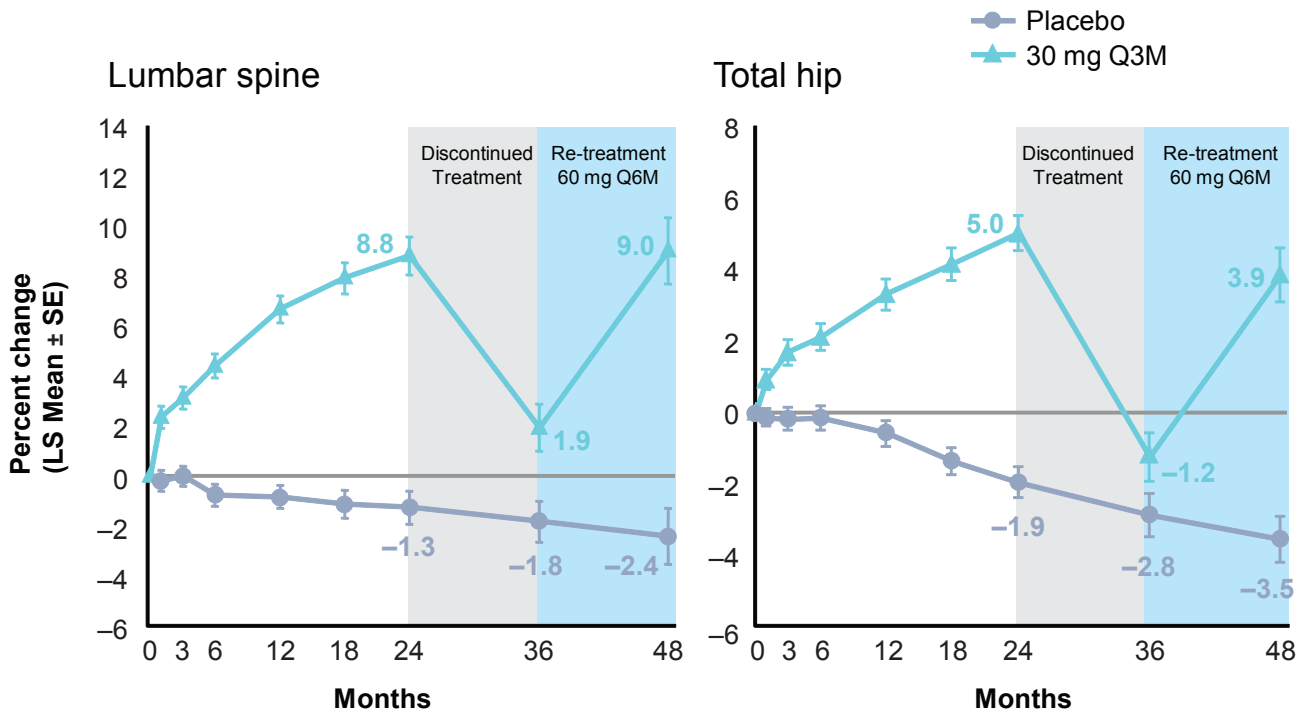

Figure 4 Effect of denosumab re-treatment and changes to lumbar spine (LS) and total hip bone mineral density. Reproduced with permission from Miller PD, Bolognese MA, Lewiecki EM, et al.Amg Bone Loss Study Group. Effect of denosumab on bone density and turnover in postmenopausal women with low bone mass after long-term continued, discontinued, and restarting of therapy: a randomized blinded phase 2 clinical trial. Bone. 2008;43(2):222-229. ${ }^{32}$ Copyright (C) 2008 Elsevier. 

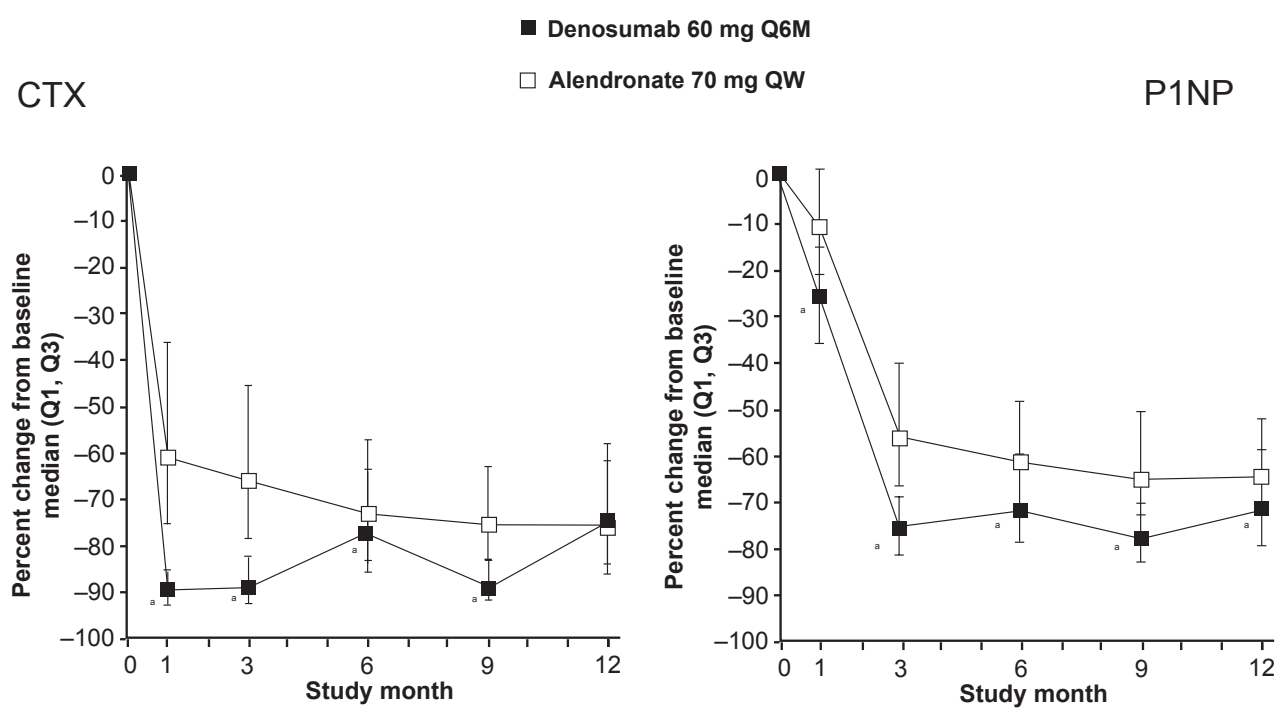

Figure 5 Effect of denusomab vs alendronate head to head trial on bone markers. Reproduced from J Bone Miner Res. 2009;24:153-161,33 with permission of the American Society for Bone and Mineral Research.

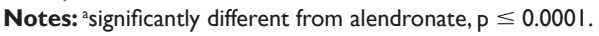

the modified Sharp erosion score was observed as early as 6 months in the 180-mg denosumab group (0.05) compared with placebo $(0.59, \mathrm{p}=0.019)$, and at 12 months, both the 60-mg (0.33) and 180-mg (0.19) denosumab groups were significantly different from the placebo group $(1.34, \mathrm{p}=0.012$ and $p=0.007$, respectively). Denosumab caused sustained suppression of BTM. There was no evidence of an effect of denosumab on joint space narrowing or measure of disease activity. Rates of AE and SAE were comparable between the denosumab and placebo groups.

\section{Metastatic bone disease}

Denosumab has been studied in patients with breast and prostate cancer and multiple myeloma. ${ }^{38-42}$ Denosumab was well tolerated and decreased markers of bone resorption, even in patients previously resistant to bisphosphonates (BP) in reducing bone resorption.

In patients with multiple myeloma $(n=25)$ or bone metastases from breast cancer $(n=29)$, a single SC dose of denosumab reduced bone resorption for at least 84 days and was well tolerated. ${ }^{38}$ The decrease in bone turnover markers was similar in magnitude but more sustained than with IV pamidronate.

In 255 women with breast cancer-related bone metastases, subcutaneous denosumab was similar to IV BP in suppressing bone turnover and reducing skeletal-related events risk. ${ }^{39}$ The safety profile was consistent with an advanced breast cancer population receiving systemic therapy.
In women with non-metastatic breast cancer and low bone mass who were receiving adjuvant aromatase inhibitor therapy, twice-yearly administration of denosumab significantly increased BMD over 24 months at trabecular and cortical bone, with overall $\mathrm{AE}$ rates similar to those of placebo. ${ }^{40}$

A next study evaluated the effects of denosumab in IV BP-naïve patients with breast cancer-related bone metastases. Denosumab suppressed bone turnover and seemed to reduce SRE risk similarly to IV BPs, with a safety profile consistent with an advanced cancer population receiving systemic therapy. ${ }^{41}$

\section{Safety of denosumab}

\section{Fracture repair}

In studies of the effect of RANKL inhibition on fracture repair, high doses of OPG given to rats at the time of fracture did not influence the formation of a normal fracture callus, but delayed callus remodeling. ${ }^{42}$ In an animal study of transverse femur fracture healing, alendronate and denosumab delayed the removal of cartilage and the remodeling of the fracture callus. ${ }^{43,44}$ However, this did not diminish the mechanical integrity of the healing fractures in mice receiving these treatments. In contrast, strength and stiffness were enhanced in these treatment groups when compared to control bones. ${ }^{44}$

\section{Immunosuppression}

As mentioned earlier, the RANKL/OPG/RANK pathway is involved in the development of the immune system. Therefore, 
safety issues about infection and neoplasm deserve special attention when therapeutically interfering with this pathway. ${ }^{2}$

OPG did not alter cellular or humoral immunity, or render mice more susceptible to bacterial challenge at doses that inhibited bone resorption. ${ }^{2}$ Furthermore, OPG prevented bone erosions and bone loss without affecting the degree of inflammation. $^{2}$

In the published clinical trials on osteoporosis, no significant differences in infections reported as SAE were found between denosumab, placebo and alendronate, ${ }^{28-33}$ except in one study in 332 subjects with osteopenia (4.9\% with denosumab versus $0.6 \%$ with placebo, $\mathrm{p}<0.05) .{ }^{34}$ The six SAEs of infections associated with denosumab in the 24-month phase- 2 study were common community-acquired infections that were successfully treated with standard antibiotics during uncomplicated hospital courses. ${ }^{32}$ The rate of infections remained unchanged from year 1 to year 2 in the denosumab group. ${ }^{32}$

No significant differences in neoplasms reported as SAE were found between denosumab, placebo and BP-treated patients. $^{38-41}$

\section{Osteonecrosis of the jaw}

Osteonecrosis of the jaw (ONJ) is a potential side effect of BPs, especially when given IV in high doses. ${ }^{45}$ The incidence of ONJ in patients using oral BPs for osteoporosis is low, and associated with other risks for ONJ, such as infection, and possibly other, not yet well defined risks and co-morbidities. ${ }^{45}$ Up until now, ONJ has not been reported in the clinical trials with denosumab.

\section{Comparison of denosumab with bisphosphonates}

The effect of RANKL inhibition is quite unique among antiresorptive agents. RANKL inhibition differed from the effects of BPs on bone in several aspects.

First, OPG, at least in animal models, does not damage the osteoclasts but decreases their number while BPs inhibit the function of osteoclasts by damaging the cytoskeleton, without affecting or even increasing the number of osteoclasts. ${ }^{29,46,47}$ Second, denosumab resulted in a quicker decrease in markers of bone resorption than alendronate. ${ }^{29}$ Third, this effect on bone resorption was more pronounced than with alendronate. ${ }^{29}$ Fourth, the effect of denosumab is completely reversible and cleared over a relatively short period of time, in contrast to the long term effects of BPs. ${ }^{28}$ The complete reversibility of bone remodeling after denosumab could result in a quicker response of changes in BMD than BPs when anabolic agents such as PTH are subsequently given. ${ }^{48}$ Fifth, denosumab effects were greater than alendronate on BMD and on strength indices derived from hip geometry evaluated by DXA at the intertrochanteric and femoral shaft sites. ${ }^{31}$ Sixth, patient preference could be different between denosumab 60 mg every 6 months and weekly bisphosphonates. ${ }^{49}$ Lastly, denosumab is the first anti-bone resorbing agent that has been shown to halt bone erosions in RA. ${ }^{37}$ Although bisphosphonates are successful in preventing erosions in animal models of inflammatory arthritis, support from studies in humans is lacking, ${ }^{50-52}$ with the exception of some effect of zoledronate that showed slowing in the number of erosions. ${ }^{53}$

\section{Conclusions}

The RANKL/RANK/OPG pathway plays a central role in coupling bone formation and resorption during normal bone turnover and a central and common role in a wide spectrum of diseases characterized by disturbed bone remodeling and increased bone resorption and destruction (osteoporosis, Paget's disease of bone, RA, metastatic bone disease). Clinical trials in postmenopausal women indicate that denosumab, a RANKL-specific recombinant humanized monoclonal antibody, is effective in suppressing bone resorption resulting in increase in BMD in osteoporosis, and has the potential to prevent progression of erosions in RA and of skeletal-related events in metastatic bone disease. The effects on fracture reduction in osteoporosis are awaited from the recently finished FREEDOM study. In clinical trials with denosumab, overall AEs and SAEs were similar to placebo, indicating a favorable safety profile in these diseases, but data on long-term safety will be needed.

\section{Disclosures}

The author discloses no conflicts of interest.

\section{References}

1. Seeman E, Delmas PD. Bone quality - the material and structural basis of bone strength and fragility. N Engl J Med. 2006;354(21):2250-2261.

2. Kearns AE, Khosla S, Kostenuik PJ. Receptor activator of nuclear factor kappaB ligand and osteoprotegerin regulation of bone remodeling in health and disease. Endocr Rev. 2008;29(2):155-192.

3. Hattner R, Epker BN, Frost HM. Suggested sequential mode of control of changes in cell behaviour in adult bone remodelling. Nature. 1965; 206(983):489-490.

4. Parfitt AM. Quantum concept of bone remodeling and turnover: implications for the pathogenesis of osteoporosis. Calcif Tissue Int. 1979; 24;28(1):1-5.

5. Simonet WS, Lacey DL, Dunstan CR, et al. Osteoprotegerin: a novel secreted protein involved in the regulation of bone density. Cell. 1997;89(2):309-319.

6. Lacey DL, Timms E, Tan HL, et al. Osteoprotegerin ligand is a cytokine that regulates osteoclast differentiation and activation. Cell. 1998;93(2):165-176. 
7. Bucay N, Sarosi I, Dunstan CR, et al. Osteoprotegerin-deficient mice develop early onset osteoporosis and arterial calcification. Genes Dev. 1998;12(9):1260-1268.

8. Rodan GA, Martin TJ. Role of osteoblasts in hormonal control of bone resorption: a hypothesis. Calcif Tissue Int. 1981;33:349-351.

9. Lacey DL, Timms E, Tan HL, et al. Osteoprotegerin ligand is a cytokine that regulates osteoclast differentiation and activation. Cell. 1998;93:165-176.

10. Yasuda H, Shima N, Nakagawa N, et al. Osteoclast differentiation factor is a ligand for osteoprotegerin/osteoclastogenesis-inhibitory factor and is identical to TRANCE/RANKL. Proc Natl Acad Sci US A. 1998;95(7):3597-3602.

11. Wong B, Josien R, Lee SY, et al. TRANCE (tumor necrosis factor [TNF]-related activation induced cytokine), a new TNF family member predominantly expressed in T cells, is a dendritic cell-specific survival factor. J Exp Med. 1997;186:2075-2080.

12. Glass DA, Bialek $P, A h n$ JD, et al. Canonical wnt signaling in differentiated osteoblasts controls osteoclast differentiation. Dev Cell. 2005; 8:751-764.

13. Tatsumi S, Ishii K, Amizuka N, et al. Targeted ablation of osteocytes induces osteoporosis with defective mechanotransduction. Cell Metab. 2007;5:464-475

14. Goldring SR, Goldring MB. Eating bone or adding it: the Wnt pathway decides. Nat Med. 2007;13(2):133-134.

15. Burgess T, Qian YX, Kaufman S, et al. The ligand for osteoprotegerin (OPGL) directly activates mature osteoclasts. J Cell Biol. 1999;45:527-53.

16. Akatsu T, Murakami T, Ono K, et al. Osteoclastogenesis inhibitory factor exhibits hypocalcemic effects in normal mice and in hypercalcemic nude mice carrying tumors associated with humoral hypercalcemia of malignancy. Bone. 1998;23:495-498.

17. Teitelbaum SL, Ross FP. Genetic regulation of osteoclast development and function. Nat Rev Genet. 2003;4(8):638-49.

18. Cleutjens KB, Faber BC, Rousch M, et al. Noninvasive diagnosis of ruptured peripheral atherosclerotic lesions and myocardial infarction by antibody profiling. J Clin Invest. 2008;118(8):2979-2985.

19. Hughes AE, Ralston SH, Marken J, et al. Mutations in TNFRSF11A, affecting the signal peptide of RANK, cause familial expansile osteolysis. Nat Genet. 2000;24(1):45-48.

20. Schett G, Redlich K, Smolen JS. The role of osteoprotegerin in arthritis. Arthritis Res Ther. 2003;5(5):239-245.

21. Pettit AR, Walsh NC, Manning C, Goldring SR, Gravallese EM. RANKL protein is expressed at the pannus-bone interface at sites of articular bone erosion in rheumatoid arthritis. Rheumatology (Oxford). 2006;45(9):1068-1076.

22. Gravallese EM, Harada Y, Wang JT, Gorn AH, Thornhill TS, Goldring SR. Identification of cell types responsible for bone resorption in rheumatoid arthritis and juvenile rheumatoid arthritis. Am J Pathol. 1998;152(4):943-951.

23. Goldring SR. Bone and joint destruction in rheumatoid arthritis: what is really happening? J Rheumatol Suppl. 2002;65:44-48. Erratum in: J Rheumatol Suppl. 2002;29(11):2471.

24. Paget $\mathrm{S}$. The distribution of secondary growths in cancer of the breast. Lancet. 1889;1:571-573.

25. Schwarz EM, Ritchlin CT. Clinical development of anti-RANKL therapy. Arthritis Res Ther. 2007;9 Suppl 1:S7.

26. Berenson JR. Advances in the biology and treatment of myeloma bone disease. Semin Oncol. 2002;29:11-16.

27. Bekker PJ, Holloway D, Nakanishi A, Arrighi M, Leese PT, Dunstan CR. The effect of a single dose of osteoprotegerin in postmenopausal women. J Bone Miner Res. 2001;16(2):348-360.

28. Bekker PJ, Holloway DL, Rasmussen AS, et al. A single-dose placebo-controlled study of AMG 162, a fully human monoclonal antibody to RANKL, in postmenopausal women. J Bone Miner Res. 2004;19(7):1059-1066.

29. McClung MR, Lewiecki EM, Cohen SB, et al. Denosumab in postmenopausal women with low bone mineral density. $N$ Engl J Med. 2006;354:821-831.
30. Lewiecki EM, Miller PD, McClung MR, et al. AMG 162 Bone Loss Study Group. Two-year treatment with denosumab (AMG 162) in a randomized phase 2 study of postmenopausal women with low BMD. J Bone Miner Res. 2007;22(12):1832-1841.

31. Beck TJ, Lewiecki EM, Miller PD, et al. Effects of denosumab on the geometry of the proximal femur in postmenopausal women in comparison with alendronate. J Clin Densitom. 2008;11(3):351-359.

32. Miller PD, Bolognese MA, Lewiecki EM, et al. Amg Bone Loss Study Group. Effect of denosumab on bone density and turnover in postmenopausal women with low bone mass after long-term continued, discontinued, and restarting of therapy: a randomized blinded phase 2 clinical trial. Bone. 2008;43(2):222-229.

33. Brown JP, Prince RL, Deal C, et al. Comparison of the effect of denosumab and alendronate on BMD and biochemical markers of bone turnover in postmenopausal women with low bone mass: a randomized, blinded, phase 3 trial. J Bone Miner Res. 2009;24(1):153-161.

34. Bone HG, Bolognese MA, Yuen CK, et al. Effects of denosumab on bone mineral density and bone turnover in postmenopausal women. J Clin Endocrinol Metab. 2008;93(6):2149-2157.

35. Cummings SR, McClung MR, Christiansen C, et al. A phase III study of the effects of denosumab on vertebral, nonvertebral, and hip fracture in women with osteoporosis: results from the FREEDOM Trial. ASBMR meeting, Montreal, 2008; Abstract 1286.

36. Geusens PP, Landewé RB, Garnero P, et al. The ratio of circulating osteoprotegerin to RANKL in early rheumatoid arthritis predicts later joint destruction. Arthritis Rheum. 2006;54(6):1772-1777.

37. Cohen SB, Dore RK, Lane NE, et al. Denosumab Rheumatoid Arthritis Study Group. Denosumab treatment effects on structural damage, bone mineral density, and bone turnover in rheumatoid arthritis: a twelve-month, multicenter, randomized, double-blind, placebo-controlled, phase II clinical trial. Arthritis Rheum. 2008;58(5): 1299-1309.

38. Body JJ, Facon T, Coleman RE, et al. A study of the biological receptor activator of nuclear factor-kappaB ligand inhibitor, denosumab, in patients with multiple myeloma or bone metastases from breast cancer. Clin Cancer Res. 2006;15;12(4):1221-1228.

39. Lipton A, Steger GG, Figueroa J, et al. Randomized active-controlled phase II study of denosumab efficacy and safety in patients with breast cancer-related bone metastases. J Clin Oncol. 2007;25(28): 4431-4437.

40. Ellis GK, Bone HG, Chlebowski R, et al. Randomized trial of denosumab in patients receiving adjuvant aromatase inhibitors for nonmetastatic breast cancer. J Clin Oncol. 2008;26(30):4875-4882.

41. Lipton A, Steger GG, Figueroa J, et al. Extended efficacy and safety of denosumab in breast cancer patients with bone metastases not receiving prior bisphosphonate therapy. Clin Cancer Res. 2008;14(20): 6690-6696.

42. Ulrich-Vinther M, Andreassen TT. Osteoprotegerin treatment impairs remodeling and apparent material properties of callus tissue without influencing structural fracture strength. Calcif Tissue Int. 2005;76:280-286.

43. Kon T, Cho TJ, Aizawa T, et al. Expression of osteoprotegerin, receptor activator of NF-_B ligand (osteoprotegerin ligand) and related proinflammatory cytokines during fracture healing. J Bone Miner Res. 2001;16:1004-1014.

44. Gerstenfeld LC, Sacks DJ, Pelis M, et al. Comparison of effects of the bisphosphonate alendronate versus the RANKL inhibitor denosumab on murine fracture healing. J Bone Miner Res. 2008 Nov 18. [Epub ahead of print].

45. Khosla S, Burr D, Cauley J, et al. American Society for Bone and Mineral Research. Bisphosphonate-associated osteonecrosis of the jaw: report of a task force of the American Society for Bone and Mineral Research. J Bone Miner Res. 2007;22(10):1479-1491.

46. Glowacki J. The deceiving appearances of osteoclasts. $N$ Engl J Med. 2009;360(1):80-82.

47. Weinstein RS, Roberson PK, Manolagas SC. Giant osteoclast formation and long-term oral bisphosphonate therapy. $N$ Engl J Med. 2009; 360(1):53-62. 
48. Geusens PP, Roux CH, Reid DM, et al. Drug Insight: choosing a drug treatment strategy for women with osteoporosis-an evidence - based clinical perspective. Nat Clin Pract Rheumatol. 2008;4(5):240-248.

49. Gold DT, Horne R, Borenstein J, Varon S, Macarios D. Preference and satisfaction with a 6-monthly subcutaneous injection versus a weekly tablet for treatment of low bone mass. Abstract M372.

50. Valleala H, Laasonen L, Koivula MK, Mandelin J, Friman C, Risteli J, et al. Two year randomized controlled trial of etidronate in rheumatoid arthritis: changes in serum aminoterminal telopeptides correlate with radiographic progression of disease. J Rheumatol. 2003;30:468-473.
51. Lodder MC, Van Pelt PA, Lems WF, Kostense PJ, Koks CH, Dijkmans BA. Effects of high dose intravenous pamidronate on disease activity and bone metabolism in patients with active rheumatoid arthritis: a randomized, double-blind, placebo-controlled trial. J Rheumatol. 2003;30:2080-2082.

52. Maksymowych WP. Bisphosphonates for arthritis: a confusing rationale. J Rheumatol. 2003;30:430-434.

53. Jarrett SJ, Conaghan PG, Sloan VS, et al. Preliminary evidence for a structural benefit of the new bisphosphonate zoledronic acid in early rheumatoid arthritis. Arthritis Rheum. 2006;54(5):1410-1414.

\section{Publish your work in this journal}

Clinical Interventions in Aging is an international, peer-reviewed journal focusing on evidence-based reports on the value or lack thereof of treatments intended to prevent or delay the onset of maladaptive correlates of aging in human beings. This journal is indexed on PubMed Central, MedLine, the American Chemical Society's 'Chemical
Abstracts Service' (CAS), Scopus and the Elsevier Bibliographic databases. The manuscript management system is completely online and includes a very quick and fair peer-review system, which is all easy to use. Visit http://www.dovepress.com/testimonials.php to read real quotes from published authors.

Submit your manuscript here: http://www.dovepress.com/clinical-interventions-in-aging-journal 